\title{
Effects of Xanthine Derivatives on Phosphatidylcholine Secretion in Primary Culture of Rat Type II Pneumocytes
}

\author{
Manabu Okumura ${ }^{1}$, Michio Tsuruoka ${ }^{1}$, Yoichiro Isohama ${ }^{2}$, Hirofumi Kai ${ }^{2}$, Kazuo Takahama ${ }^{2}$ and Takeshi Miyata ${ }^{2, *}$ \\ ${ }^{1}$ Department of Pharmacy, Miyazaki Medical College, 5200 Kihara, Kiyotake-machi, Miyazaki 889-16, Japan \\ ${ }^{2}$ Department of Pharmacological Sciences, Faculty of Pharmaceutical Sciences, Kumamoto University, 5-1 Oe-honmachi, Kumamoto 862, Japan
}

Received October 21, 1994 Accepted December 5, 1994

\begin{abstract}
Xanthine derivatives, pentoxifylline, aminophylline, theophylline and chinoin-170, increased phosphatidylcholine secretion in a primary culture of rat type II pneumocytes. However, these xanthines alone had no effect on intracellular cAMP levels in type II pneumocytes. In contrast, terbutaline-induced secretion of phosphatidylcholine was augmented by these xanthines, and the augmentation depended on the increase of cAMP levels. These results suggest that the xanthines induce phosphatidylcholine secretion possibly through cAMP-dependent and cAMP-independent pathways in the primary culture of rat type II pneumocytes.
\end{abstract}

Keywords: Xanthine, Type II pneumocyte, Phosphatidylcholine secretion

Lung surfactant, the phospholipid-rich material lining the alveolar surface, is synthesized and stored in type II pneumocytes and secreted by exocytosis $(1-3)$. Secretion of surfactant phospholipids has been shown to be influenced by a variety of physiological and pharmacological agents $(1,3)$. $\beta$-Adrenergic agonists and forskolin increase intracellular cAMP, activate cAMP-dependent kinases and then stimulate pulmonary surfactant secretion. Xanthine derivatives such as theophylline inhibit cAMP phosphodiesterase and increase intracellular cAMP level. It seems likely that xanthine derivatives induce surfactant secretion through the increase of cAMP. One finding showed that isobutylmethylxanthine, a phosphodiesterase inhibitor, and 8-bromo cAMP, but not 8bromo cGMP, stimulated pulmonary surfactant secretion (4). However, they did not examine the relationship between stimulation of surfactant secretion by xanthine derivatives and the increase of cAMP level.

In the present study, to determine whether xanthine derivatives can induce surfactant secretion through an increase of cAMP level, we examined the effects of several xanthine derivatives on secretion of phosphatidylcholine, a major lipid component in lung surfactant, in primary cultures of rat type II pneumocytes.

Type II pneumocytes were isolated from the lungs of adult specific-pathogen-free male Wistar rats $(180-200 \mathrm{~g})$

\footnotetext{
* To whom correspondence should be addressed.
}

according to the method of Dobbs et al. (5). This method routinely yielded $10^{7}$ cells per rat. The cells were suspended at $10^{6}$ cells $/ \mathrm{ml}$ in Dulbecco's modified Eagle's medium supplemented with $10 \%$ fetal bovine serum, $74 \mathrm{kBq} / \mathrm{ml}$ [methyl ${ }^{3} \mathrm{H}$ ]choline (specific activity, $3.0 \mathrm{TBq} / \mathrm{mmol}$ ), 100 units $/ \mathrm{ml}$ penicillin and $100 \mu \mathrm{g} / \mathrm{ml}$ streptomycin; plated on 24-well tissue culture plate (Falcon 3047); and then cultured at $37^{\circ} \mathrm{C}$ in $5 \% \mathrm{CO}_{2}$-air for $18 \mathrm{hr}$. Non-adherent cells were removed from the wells by washing before the assay. The purity of the type II pneumocytes monolayer was $95 \pm 3 \%$. For cellular identification, the sample was stained with a tannic acid and polychrome stain and alkaline phosphatase stain. The viability of type II pneumocytes was $98 \pm 2 \%$ as judged by the trypan blue exclusion test.

Secretion of phosphatidylcholine by cultured type II pneumocytes was determined as follows: The cells were rinsed with fresh serum- and antibiotics-free medium to remove $\left[{ }^{3} \mathrm{H}\right]$ choline and unattached cells; the test agents were added after $30 \mathrm{~min}$ and the incubation was continued for $90 \mathrm{~min}$. At the end of the incubation period, the medium was aspirated off, the cells were lysed with 2 $\mathrm{ml}$ ice-cold $0.05 \%$ Triton X-100 solution and lipids were extracted from both cells and medium with chloroform and methanol $(2: 1, \mathrm{v} / \mathrm{v})$. Phosphatidylcholine was separated from the other phospholipids by thin-layer chromatography (6), and its radioactivity was measured with a liquid scintillation counter after the addition of $5 \mathrm{ml}$ 
Aquasol II to each sample. Secretion was expressed as the amount of $\left[{ }^{3} \mathrm{H}\right]$ phosphatidylcholine in the medium after the 90-min incubation, as a percentage of that in cells plus medium.

For cAMP measurement, the medium was aspirated at various times after the addition of test agents and the cells extracted with $0.1 \mathrm{~N} \mathrm{HCl}$. The extracts were immediately frozen, lyophilized and stored at $-70^{\circ} \mathrm{C}$. The sample was reconstituted and acetylated, and the cAMP content was determined as described by the radioimmunoassay kit manufacturer.

To assess cellular integrity, the activity of lactate dehydrogenase ( $(\mathrm{DH})$ in the cells and medium was measured with an LDH kit-s (Nippon Shoji Co., Ltd., Osaka). The LDH activity released into the medium did not exceed $1 \%$ of the total cell content in all experiments.

Statistical analyses were perfomed by means of Duncan's multiple-range test.

The rats were purchased from Kyudo Farm (Fukuoka), tissue culture medium from Nissui Pharmaceutical Co., Ltd. (Tokyo) and fetal bovine serum from Sera-Lab Ltd. (Sussex, England). [Methyl- ${ }^{3} \mathrm{H}$ ]choline, Aquasol II and cyclic AMP radioimmunoassay kits were obtained from NEN Research Products (Boston, MA, USA). 3,7-Dihydro-1,3-dimethyl-7-[(5-methyl-1,2,4-oxadiazol-3yl)methyl] $1 H$-purine-2,6-dione (chinoin-170) was from Fujisawa Pharmaceutical Co., Ltd. (Osaka), and the other reagents and biochemicals were from Sigma Chemicals Co. (St. Louis, MO, USA). All xanthine derivatives, except for theophylline in dimethyl sulfoxide, were dissolved in distilled water at $100 \mathrm{mM}$ and diluted to each concentration, and then $10 \mu \mathrm{l}$ of each xanthine was added into $1 \mathrm{ml}$ of culture media in each well. In preliminary experiments, $1 \%$ dimethyl sulfoxide did not differ from distilled water in phosphatidylcholine secretion (data not shown). Therefore, in the following experiments, we used distilled water in a control group.

First, to determine whether xanthine derivatives can induce phosphatidylcholine secretion, we examined the effects of pentoxifylline, aminophylline, theophylline, and a novel xanthine derivative, chinoin-170 (7), at the effective doses of xanthine derivatives as previously reported. Those effective doses in smooth muscle relaxation were $10^{-3}$ to $10^{-4} \mathrm{M}(8)$. We used this relatively high concentration of xanthine derivatives. All xanthine derivatives used here increased phosphatidylcholine secretion (Table 1). The increased secretions were augmented in combination with terbutaline $\left(10^{-6} \mathrm{M}\right)$, a $\beta_{2}$-adrenergic receptor agonist (Table 1).

Next, to see the relationship between the phosphatidylcholine secretion and intracellular cAMP levels, we measured the amount of intracellular cAMP in type II pneumocytes. Terbutaline increased the cAMP level within $5 \mathrm{~min}$ after its addition (Fig. 1). Xanthine derivatives alone did not affect the cAMP levels even at the dose in which the secretion of phosphatidylcholine was increased. However, xanthine derivatives in combination with terbutaline $\left(10^{-6} \mathrm{M}\right)$ increased cAMP levels more than terbutaline alone did (Fig. 1).

We have shown that all xanthine derivatives used here induced phosphatidylcholine secretion. However, xanthine derivatives alone had no effect on the cAMP levels.

Table 1. Effects of xanthine derivatives on phosphatidylcholine secretion in primary culture of rat type II pneumocytes

\begin{tabular}{|c|c|c|c|}
\hline & & $\%$ Secreted & $\%$ Control \\
\hline Control & & $1.30 \pm 0.14$ & 100 \\
\hline Terbutaline & $\left(10^{-6} \mathrm{M}\right)$ & $2.08 \pm 0.06^{a}$ & 160 \\
\hline \multirow[t]{4}{*}{ Pentoxifylline } & $\left(10^{-5} \mathrm{M}\right)$ & $1.84 \pm 0.04^{\mathrm{a})}$ & 142 \\
\hline & $\left(10^{-4} \mathrm{M}\right)$ & $2.03 \pm 0.04^{\mathrm{a})}$ & 156 \\
\hline & $\left(10^{-3} \mathrm{M}\right)$ & $2.74 \pm 0.07^{\mathrm{a}}$ & 211 \\
\hline & $\left(10^{-3} \mathrm{M}\right)+$ Terbutaline $\left(10^{-6} \mathrm{M}\right)$ & $3.47 \pm 0.15^{\mathrm{a}, \mathrm{b})}$ & 267 \\
\hline \multirow[t]{4}{*}{ Aminophylline } & $\left(10^{5} \mathrm{M}\right)$ & $1.68 \pm 0.12$ & 129 \\
\hline & $\left(10^{-4} \mathrm{M}\right)$ & $1.81 \pm 0.13^{\mathrm{a})}$ & 139 \\
\hline & $\left(10^{-3} \mathrm{M}\right)$ & $2.04 \pm 0.05^{\mathrm{a}}$ & 157 \\
\hline & $\left(10^{-3} \mathrm{M}\right)+$ Terbutaline $\left(10^{-6} \mathrm{M}\right)$ & $4.09 \pm 0.06^{\mathrm{a}), \mathrm{b}}$ & 315 \\
\hline \multirow[t]{2}{*}{ Theophylline } & $\left(10^{-3} \mathrm{M}\right)$ & $2.32 \pm 0.06^{\mathrm{a}}$ & 178 \\
\hline & $\left(10^{3} \mathbf{M}\right)+$ Terbutaline $\left(10^{-6} \mathrm{M}\right)$ & $3.33 \pm 0.15^{a), b)}$ & 256 \\
\hline \multirow[t]{2}{*}{ Chinoin-170 } & $\left(10^{-3} \mathrm{M}\right)$ & $1.82 \pm 0.11^{\mathrm{a})}$ & 140 \\
\hline & $\left(10^{-3} \mathrm{M}\right)+$ Terbutaline $\left(10^{-6} \mathrm{M}\right)$ & $3.19 \pm 0.33^{\mathrm{a}), \mathrm{b})}$ & 245 \\
\hline
\end{tabular}

Each value is the mean \pm S.E. of 5 experiments. ")Significantly different at $\mathbf{P}<0.05$ vs control,

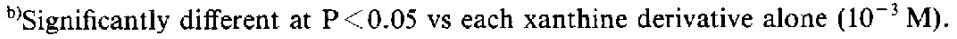




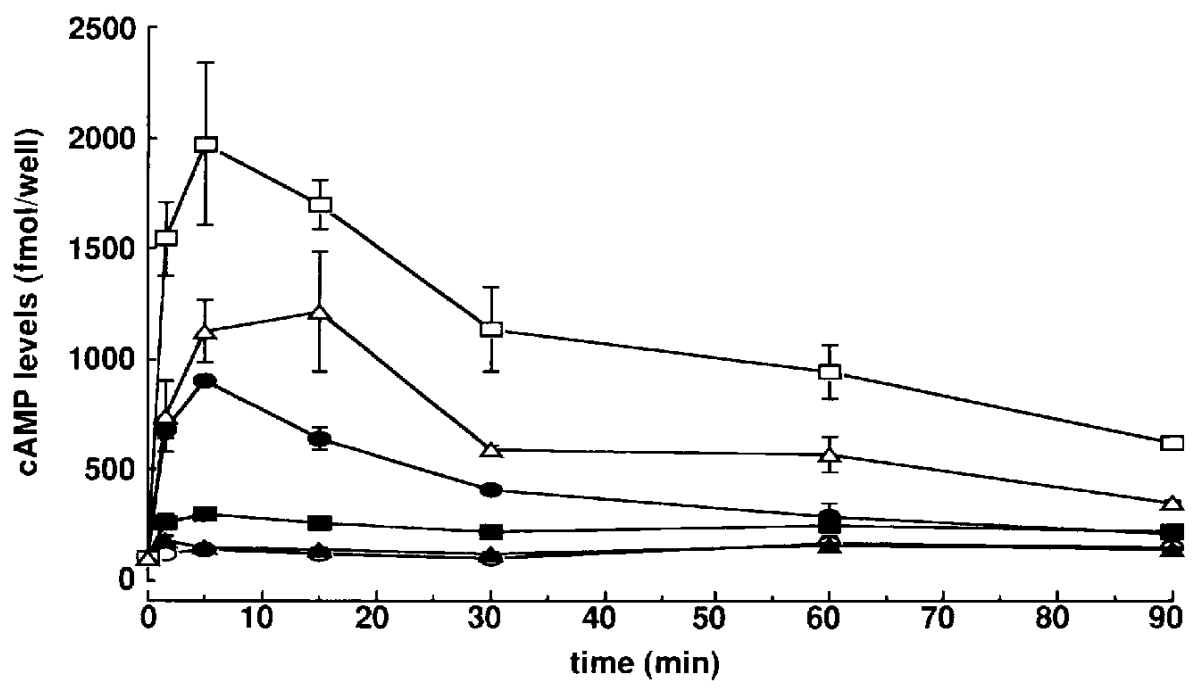

Fig. 1. Effects of xanthine derivatives $\left(10^{-3} \mathrm{M}\right)$ in combination with terbutaline $\left(10^{6} \mathrm{M}\right)$ on cAMP level of type II pneumocytes. The cells were incubated in the presence or absence of agents for the periods indicated, after which cellular cAMP was extracted and measured as described in the text. The data are the mean \pm S.E. (bar) from 4 experiments. $Q$, control; $O$, terbutaline; $\boldsymbol{\square}$, pentoxifylline; $\boldsymbol{\Delta}$, theophylline; $\square$, terbutaline + pentoxifylline; $\Delta$, terbutaline + theophylline.

It seems that there is no correlation between phosphatidylcholine secretion and the cAMP levels. This result is consistent with the finding in tracheal muscle relaxation. There was a positive correlation between the $\mathrm{EC}_{50}$ of xanthine derivatives for tracheal muscle relaxation and the $\mathrm{IC}_{50}$ for phosphodiesterase inhibition, but there was no obvious correlation with the increase in intracellular cAMP in tracheal muscle (8).

On the other hand, the data for the combination of xanthine derivatives with terbutaline indicated that there was some positive relationship between phosphatidylcholine secretion increased by xanthine derivatives and the cAMP levels. Our interpretation for the discrepancies is that xanthine derivatives have two mechanisms to induce phosphatidylcholine secretion, cAMP-independent and cAMP-dependent mechanisms. Xanthine derivatives possess various effects in addition to phosphodiesterase inhibition, such as inhibition of intracellular calcium mobilization and adenosine receptor blocking (9). Of the xanthine derivatives used here, pentoxifylline may have the highest activity of phosphatidylcholine secretion (Table 1). Pentoxifylline has been demonstrated to inhibit neutrophil activation. The possible mechanism for this inhibitory effect is that pentoxifylline can affect both the cytoskeletal architecture of unstimulated neutrophils and the activation and responses of neutrophils that involve actin polymerization and receptor-cytoskeletal interactions (10). The effect of xanthines on phosphatidylcholine secretion through a cAMP-independent pathway may be related to changes in cytoskeletal architecture of type II pneumocytes, because cytoskeletal change can induce phosphatidylcholine secretion in type II pneumocytes (3).

We used relatively high concentrations of xanthine derivatives to induce phosphatidylcholine secretion in type II pneumocytes. One reasonable explanation for this high dose is presumably that adenyl cyclase activity in isolated type II pneumocytes may be very low without any stimulants. In turn, in whole animals, xanthine derivatives may induce phosphatidylcholine secretion at the lower concentration because phosphatidylcholine secretion as well as adenyl cyclase in type II pneumocytes is constitutively activated by adrenergic nerves.

In conclusion, our study suggests that xanthine derivatives induce phosphatidylcholine secretion possibly through cAMP-dependent and cAMP-independent pathways in the primary culture of rat type II pneumocytes.

\section{REFERENCES}

1 Chander A and Fisher AB: Regulation of lung surfactant secretion. Am J Physiol 258, 241 - 253 (1990)

2 Haagsman HP and van Golde LM: Synthesis and assembly of lung surfactant. Annu Rev Physiol 53, 441 - 464 (1991)

3 Wright JR and Dobbs LG: Regulation of pulmonary surfactant secretion and clearance. Annu Rev Physiol 53, 395-414 (1991)

4 Dobbs LG and Mason RJ: Pulmonary alveolar type II cells isolated from rats. Release of phosphatidylcholine in response to beta adrenergic stimulation. J Clin Invest 63, 378-387 (1979)

5 Dobbs LG, Gonzales $R$ and Williams MC: An improved method for isolating type II cells in high yield and purity. Am Rev Respir Dis 134, $141-145$ (1986)

6 Miyata T, Kai H, Furusawa K, Nakamura H, Saito M, Okano 
$\mathrm{Y}$ and Takahama K: Secretomotor and mucolytic effects of mabuterol, a novel bronchodilator. Arch Int Pharmacodyn Ther 288, 147-160 (1987)

7 Saano V, Minker E, Joki S, Virta P, Nuutinen J and Korbonits D: Influence of chinoin-170, a novel antitussive, on the mucociliary activity in respiratory airways of rats, rabbits, guinea pigs and man. J Pharm Pharmacol 45, 799-802 (1993)

8 Bryson SE and Rodger IW: Effects of phosphodiesterase inhibitors on normal and chemically-skinned isolated airway smooth muscle. Br J Pharmacol 92, 673-681 (1987)

9 Sawynok J and Yaksh TL: Caffeine as an analgesic adjuvant: A review of pharmacology and mechanisms of action. Pharmacol Rev 45, 43-85 (1993)

10 Rao KMK, Crawford J, Currie MS and Cohen HJ: Actin depolymerization and inhibition of capping induced by pentoxifylline in human lymphocytes and neutrophils. I Cell Physiol 137, $577-582(1988)$ 